\title{
НЕСОВПАДЕНИЕ СВЯЗЕЙ ОДНИХ И ТЕХ ЖЕ ГОРМОНАЛЬНО-МЕТАБОЛИЧЕСКИХ ПАРАМЕТРОВ С РИСКОМ РАЗВИТИЯ ОСНОВНЫХ НЕИНФЕКЦИОННЫХ ЗАБОЛЕВАНИЙ: ГОРМОНОЗАВИСИМЫЕ ОПУХОЛИ В СРАВНЕНИИ С КАРДИО- И ЦЕРЕБРОВАСКУЛЯРНОЙ ПАТОЛОГИЕЙ
}

\author{
Берштейн Л.М.
}

\author{
НМИЦ онкологии им. Н.Н.Петрова МЗ РФ, Санкт-Петербург
}

\begin{abstract}
АКТУАЛЬНОСТЬ, ЦЕЛИ И ЗАДАЧИ: ТаКИе ОПУХОЛИ Как рак эндОМетрИя, МОЛОчной Железы, простаты И др. составляют немалую долю всех новообразований и анализируются с позиций взаимоотношений с иными основными хроническими неинфекционными заболеваниями/ОН3 (в частности, кардиоваскулярными и цереброваскулярными), а также факторами риска последних, в том числе, гормонально-метаболической природы. Точка зрения об определенном сходстве этих факторов при ОНЗ как онкологического, так и неонкологического типа, нередко поддерживается, но не всегда подтверждается, примеры чего могут быть найдены ниже.
\end{abstract}

\section{РЕЗУЛЬТАТЫ.}

Пример 1. Вес новорожденных Вес плода при рождении рассматривается как один из факторов, предсказывающих в определенной степени спектр заболеваемости не только самого новорожденного, но и его матери. Так, риск сердечно-сосудистых заболеваний у матерей выше в том случае, если они рожали детей со сниженной массой тела, а риск онкозаболеваемости повышается у женщин, родивших потомство с большой массой тела при рождении, в частности, >4000 г (Berstein L. 2013 Womens Health (Lond). 9(4): 361-371).

Пример 2. Рост во взрослом возрасте Имеющаяся литература свидетельствует, что чем выше рост взрослых мужчин и женщин, тем больше смертность от злокачественных новообразований и меньше - от ишемической болезни сердца и инсульта (Stefan N. et al., 2016, Lancet Diabetes Endocrinol. 4(5): 457-467). Вдобавок, люди с более высоким ростом реже демонстрируют признаки инсулинорезистентности и сахарного диабета 2 типа (СД2).

Пример 3. Сахарный диабет в семье Среди факторов риска СД2 особенно часто упоминаются избыточная масса тела, недостаточная физическая активность и семейная история диабета, в том числе в варианте без явной генетической подкладки. Хотя можно было бы ожидать, что повышая риск СД2, семейный диабет мог бы способствовать и повышению частоты распространения онкологических заболеваний, по ряду опубликованных данных, наблюдается тенденция к снижению частоты последних (Hemminki K. et al.,2010. Oncologist, 15(6):548-555) в противоположность риску развития кардиоваскулярной патологии (Bonnet F et al. 2016 Diabetes Metab. 42(3): 139-141)

Согласно современной классификации ProMisE (Talhouk A et al Cancer 2017; 123: 802-813) в настоящее время выделяют четыре молекулярно-биологических типа рака эндометрия (РЭ), которые нуждаются в дополнительном изучении, включая гормонально-метаболические и ассоциированные с ними особенности не только опухолевой ткани, но и заболевающих женщин. По предварительным данным, накопленным нами при обследовании больных РЭ с различными типами этой опухоли, выяснилось, что у больных с мутацией гена POLE ниже как частота явного, так и семейного диабета. Реже выявляется семейный диабет и у больных с гиперэкспрессией онкобелка $p 53$, что несвойственно пациенткам с дефектом репарации ошибочно спаренных нуклеотидов (MMR-D) в опухолевой ткани, а также больным без характерного молекулярного профиля новообразования (Берштейн и соавт. Вопр. онкол. 2018, 64(3): 394-398). При этом в последнем случае не выявилось отличий между подгруппами с индексом массы тела >30.0 и<30.0 по уровню эстрадиола и инсулина, несмотря на повышение в первой из них отношения лептин/ адипонектин за счет снижения адипонектинемии - неоднозначного маркера риска и прогрессирования кардиоваскулярной патологии (L.Woodward et al. Br J Pharmacol. 2017, 174(22): 4007-4020).

Выводы: Представленные сведения в совокупности подтверждают предположение о «расщеплении»/дихотомии отдельных ОН3 (имея в виду, в частности, онкологическую и неонкологическую патологию) по их «ответу» на влияние одних и тех же гормонально-метаболических факторов риска, что нуждается в дальнейшем анализе, в том числе, и применительно к способам воздействия на подобные, не совпадающие по своим итогам последствия.

Работа частично поддержана грантом РФФИ 18-015-00026. 